Pacific Journal of Mathematic 


\title{
ON TWO CONGRUENCES FOR PRIMALITY
}

\author{
M. V. Subbarao
}

\section{In this paper we consider the congruences}

$$
n \sigma(n) \equiv 2(\bmod \varphi(n)), \varphi(n) t(n)+2 \equiv 0(\bmod n) .
$$

1. Introduction. Apart from the classical Wilson's theorem (that a positive integer $p>1$ is a prime if and only if $(p-1) !+$ $1 \equiv 0(\bmod p))$ and its variants and corollaries, there is probably no other simple primality criterion in the literature in the form of a congruence. In this connection, we may recall Lehmer's congruence [1]:

$$
n-1 \equiv 0 \bmod \phi(n) .
$$

This is satisfied by every prime. We do not yet know if it has any composite $n$ as a solution. In 1932, Lehmer [1] showed that if there exists a composite number $n$ satisfying (1.1), then $n$ must be odd and square free and have at least seven distinct prime factors. This result was improved in 1944 by Fr. Schuh [4] who showed that such a $n$ must have at least eleven prime factors. In 1970, E. Lieuwens [2] corrected an error in the proof of Schuh.

In the congruences we shall consider,

$$
n \sigma(n) \equiv 2(\bmod \phi(n))
$$

and

$$
\phi(n) t(n)+2 \equiv 0(\bmod n),
$$

where $\phi(n)$ is Euler's totient, and $t(n)$ and $\sigma(n)$ are respectively the number and sum of the divisors of $n$. Each of these is satisfied whenever $n$ is a prime. It is a simple matter to solve (1.2) completely (Theorem 1). However, the problem of solving (1.3) for all composite integers $n$ seems to be a deep one, and we offer only a partial solution.

2. THEOREM 1. The only composite numbers $n$ satisfying (1.2) are $n=4,6$, and 22 .

Proof. Let a solution of (1.2) be

$$
n=2^{a} p_{1}^{a_{1}} \cdots p_{r}^{a}
$$

where $p_{1}, \cdots, p_{r}$ are the distinct odd prime divisors of $n$. If for some $i(1 \leqq i \leqq r), a_{i}>1$, then $p_{i} \mid \phi(n)$ and $p_{i} \mid n$, so that $p_{i} \mid 2$, an absurdity. Hence 


$$
a_{1}=a_{2}=\cdots=a_{r}=1 .
$$

An analogous argument shows that $a=0,1$ or 2 . Hence $n=$ $2^{a} p_{1} p_{2} \cdots p_{r}$, where $a=0,1$ or 2 . Next, when $n$ is in this form, $2^{r} \mid \sigma(n)$ and $2^{r} \mid \phi(n)$, so that we should have $2^{r} \mid 2$, on using the congruence. Hence $r=0$ or 1 , and we get $n=2,4, p_{1}, 2 p_{1}, 4 p_{1}$ for the possible solutions of (1.2). However, $n=4 p_{1}$ is impossible, for otherwise $4 \mid \phi(n)$, and this would imply, on using the congruence, that $4 \mid 2$.

In the next place, if $n=2 p_{1}$, we have

$$
6 p_{1}\left(p_{1}+1\right) \equiv 2 \bmod \left(p_{1}-1\right) .
$$

This shows that $\left(p_{1}-1\right) \mid 10$, and this gives $p_{1}=2,3$, and 11 . Hence all the possible composite solutions of (1.2) are $n=4,6$, and 22 , and these are indeed solutions of the congruence.

3. The solution of congruence (1.3). Up to 100,000 , the only composite solution of (1.3) is $n=4$, and the question naturally arises if there is any composite solution $>4$. While this is still open, we devote the rest of the paper to obtain some information about such a solution if it exists.

THEOREM 2. Every composite solution $n>4$ of the congruence (1.3) satisfies the following conditions:

(A) $n$ is square-free.

(B) If $p$ is an odd prime divisor of $n$, then there is no prime divisor of the form $p x+1$.

(C) Let $K$ be defined by the relation

$$
\phi(n) t(n)+2=K n .
$$

Then $K$ and $n$ are of opposite parity and $4 \nmid K$.

(D) If $n=m$ is a solution of (1.3), then $n=2 m$ is not a solution.

Proof. For an odd prime $p$, if $p^{2} \mid n$, then $p \mid \phi(n)$; hence on using (1.2), $p \mid 2$, which is absurd. Again if $4 \mid n$ and $n>4$, a simple argument shows that (1.3) is impossible. This establishes result (A). The proofs of (B), (C), and (D) are equally easy.

LEMma. For a given $r$, the number of solutions $n$ of (2.11) having $r$ prime divisors is finite. In fact, if $p_{1}, p_{2}, \cdots, p_{r}$ are the prime divisors of $n$ in increasing order of magnitude, and if

$$
Q_{r}=\left(1-\frac{1}{2}\right)\left(1-\frac{1}{3}\right) \cdots\left(1-\frac{1}{q_{r}}\right)
$$


where $q_{r}$ is the $r$ th prime in the sequence of primes $2,3,5, \cdots\left(q_{1}=\right.$ $2, q_{2}=3$ etc.), then

$$
\begin{gathered}
2^{r} Q_{r} \leqq K \leqq 2^{r}, \\
p_{1}<r\left(1-\frac{K}{2^{r}}\right)^{-1},
\end{gathered}
$$

and for $i=2,3, \cdots, r$,

$$
p_{i-1}<p_{i}<(r-i+1)\left(1-\frac{K}{2^{r}}-\frac{1}{p_{1}}-\cdots-\frac{1}{p_{i-1}}\right)^{-1} .
$$

Proof. The relation (3.1) gives

$$
\begin{aligned}
K & =\frac{\phi(n) t(n)}{n}+\frac{2}{n} \\
& \leqq t(n)+\frac{2}{n},
\end{aligned}
$$

for $n>2$. Hence $K \leqq t(n)$. Since by Theorem $2, n$ is square free, $n=p_{1}, p_{2}, \cdots, p_{r}$, so that $t(n)=2^{r}$. Hence $K \leqq 2^{r}$.

In the next place,

$$
\begin{aligned}
K & >2^{r} \frac{\phi(n)}{n} \\
& =2^{r} \prod_{i=1}^{r}\left(1-\frac{1}{p_{i}}\right) \geqq 2^{r} Q_{r} .
\end{aligned}
$$

This completes the proof of (3.3). To prove (3.4), we note that

$$
\begin{aligned}
K & >2^{r} \frac{\phi(n)}{n}=2^{r} \prod_{i=1}^{r}\left(1-\frac{1}{p_{i}}\right) \\
& >2^{r}\left(1-\frac{1}{p_{1}}-\cdots-\frac{1}{p_{r}}\right) .
\end{aligned}
$$

Hence,

$$
1-\frac{K}{2^{r}}<\frac{1}{p_{1}}+\cdots+\frac{1}{p_{r}}<\frac{r}{p_{r}},
$$

and this gives

$$
p_{1}<r\left(1-\frac{K}{2^{r}}\right)^{-1}
$$

Again, using

$$
\frac{1}{p_{1}}+\frac{1}{p_{2}}+\cdots+\frac{1}{p_{r}}<\frac{1}{p_{1}}+\frac{r-1}{p_{2}}
$$


and proceeding as before, we get

$$
p_{1}<p_{2}<(r-1)\left(1-\frac{K}{2^{r}}-\frac{1}{p_{1}}\right)^{-1} .
$$

Continuing this process, we obtain

$$
p_{2}<p_{3}<(r-2)\left(1-\frac{K}{2^{r}}-\frac{1}{p_{1}}-\frac{1}{p_{2}}\right)^{-1},
$$

and finally,

$$
p_{r-1}<p_{r}<\left(1-\frac{K}{2^{r}}-\frac{1}{p_{1}}-\cdots-\frac{1}{p_{r-1}}\right)^{-1} .
$$

This establishes (3.4).

For a given $r,(3.3)$ shows that $K$ can take only a finite number of values, and (3.4)-(3.7) show that $p_{1}, p_{2}, \cdots, p_{r}$ can take only a finite number of values. Thus for a given $r$, the congruence (1.3) has got only a finite number of solutions, since for a given $r$ the upper and lower bounds for $K, p_{1}, p_{2}, \cdots, p_{r}$ are fixed by the relations (3.3) and (3.4). The actual solutions corresponding to any given $r$ can be obtained after a finite number of trials. Following this method, we have obtained the following results. (The details of the numerous computations involved in the proofs of Theorems 3 and 4 below are available with the authors.)

THEOREM 3. Any composite solution $n>4$ of (1.3) must have at least 4 distinct odd prime factors.

THEOREM 4. For the congruence (1.3) we have the following:

(3.8) If $K=1$ or $3 \leqq K \leqq 14$, there are no solutions.

(3.9) If $K=2$, the only solutions are all the primes and 4.

(3.10) If $K=15$, then $r=4$ or 5 .

(3.11) If $17 \leqq K \leqq 29$, then $r=5$.

(3.12) If $K=30$ or 31 , then $r=5$ or 6 .

(3.13) If $33 \leqq K \leqq 58$, then $r=6$.

(3.14) If $59 \leqq K \leqq 63$, then $r=6$ or 7 .

(3.15) If $65 \leqq K \leqq 116$, then $r=7$.

(3.16) If $117 \leqq K \leqq 127$, then $r=7$ or 8 .

(3.17) If $129 \leqq K \leqq 230$, then $r=8$.

(3.18) If $231 \leqq K \leqq 255$, then $r=8$ or 9 .

(3.19) If $257 \leqq K \leqq 457$, then $r=9$.

(3.20) If $458 \leqq K \leqq 551$, then $r=9$ or 10 .

(3.21) If $513 \leqq K \leqq 909$, then $r=10$.

(3.22) If $910 \leqq K \leqq 1023$, then $r=10$ or 11 . 
Proof. We illustrate the proof for the case when $n$ is odd. Using the lemma, we have

$$
\begin{aligned}
2^{r} \geqq K & >2^{r}\left(1-\frac{1}{p_{1}}\right)\left(1-\frac{1}{p_{2}}\right) \cdots\left(1-\frac{1}{p_{r}}\right) \\
& >2^{r}\left(1-\frac{1}{3}\right)\left(1-\frac{1}{5}\right)\left(1-\frac{1}{17}\right)\left(1-\frac{1}{23}\right) \cdots\left(1-\frac{1}{p_{r}}\right),
\end{aligned}
$$

on using part (B) of Theorem 2 and Theorem 3. Giving $K$ successive integral values and examining the consistency of the resulting inequalities while keeping in view the restrictions of Theorem 2, we get the results of the theorem.

REMARK. Any solution $n$ of (3.1) satisfies the relation

$$
2^{r}<\frac{6480}{19019} K e^{r} \log x\left(1+\log ^{-2} x\right)
$$

where $\gamma$ is Euler's constant, $r$ is the number of distinct prime factors of $n$ and $x=q_{r+5}$. To show this, we note that

$$
\begin{aligned}
& 2^{r}=t(n)<K \frac{n}{\phi(n)} \\
& <K\left(1-\frac{1}{3}\right)^{-1}\left(1-\frac{1}{5}\right)^{-1}\left(1-\frac{1}{17}\right)^{-1}\left(1-\frac{1}{23}\right)^{-1} \prod_{10 \leqq i \leqq r+5}\left(1-\frac{1}{q_{i}}\right)^{-1},
\end{aligned}
$$

on using Theorems 2 and 3. Hence

$$
2^{r}<K \cdot \frac{1}{2} \cdot \frac{6}{7} \cdot \frac{10}{11} \cdot \frac{12}{13} \cdot \frac{18}{19} \cdot Q_{r+5}^{-1}
$$

where $Q_{r+5}$ is defined as in (3.2). We now use the estimate given by Rosser and Schoenfeld [3, Theorem 8, Corollary 1] for $Q_{r+5}^{-1}$, namely $Q_{r+5}^{-1}<e^{r} \log x\left(1+\log ^{-2} x\right)$, where $x=q_{r+5}$; and obtain the stated result.

In the next theorem, $q_{u}$ denotes, as already noted, the $u$ th prime in the sequence of primes $q_{1}=2, q_{2}=3, \cdots$.

TheOREM 5. Let $K$ and $m$ be given and let $q_{u}$ be the smallest prime factor of $n$ which is a solution of the simultaneous equations

$$
\begin{gathered}
\phi(n) t(n)+2=K n \\
t(n)=m K .
\end{gathered}
$$

Then $n$ has a prime factor at least as large as

$$
q_{u}^{m}+O\left(u^{m} \exp -\log ^{b} u\right)
$$

where $b$ is any number $<3 / 5$. 
Proof. By Theorem 2, $n$ is square free. Let it have $r$ distinct prime divisors.

Then A. Walfisz [5, Satz 4, p. 187] has shown that if $\pi(x)$ denotes, as usual, the number of primes $\leqq x$, and

$$
\operatorname{li} x=\int_{2}^{x} \frac{d t}{\log t}
$$

then

$$
\pi(x)=l i(x)+O\left(x\left\{\exp -A \log ^{3 / 5} x(\log \log x)^{-1 / 5}\right\}\right),
$$

where $A$ is a positive constant. It follows that

$$
\pi(x)=l i(x)+O\left(x \exp -\log ^{a} x\right)
$$

for all $a<3 / 5$. By using a standard argument, we can show that

$$
\sum_{q \leqq x} \frac{1}{q}=\log \log x+c+O\left(\exp -\log ^{a} x\right),
$$

$q$ varying over primes.

It follows that

$$
\begin{aligned}
\sum_{q \leq x}-\log \left(1-\frac{1}{q}\right) & =\sum_{q \leq x} \frac{1}{q}+\sum_{q}\left\{-\log \left(1-\frac{1}{q}\right)-\frac{1}{q}\right\}+O\left(\frac{1}{x}\right) \\
& =\log \log x+c+O\left(\exp -\log ^{a} x\right)
\end{aligned}
$$

for all $a<3 / 5$, where $c$ is an absolute constant (not necessarily the same as the $c$ used before).

Hence for any given $h$ for which $h=O\left(x^{m}\right)$, we have

$$
\begin{aligned}
& \sum_{x \leqq q \leqq x}-\log \left(1-\frac{1}{q}\right) \\
& \quad=\log \log \left(x^{m}+h\right)-\log \log x+O\left(\exp -\log ^{a} x\right)
\end{aligned}
$$

for all $a<3 / 5$. If we choose $h=x^{m} \exp \left(-\log ^{b} x\right)$, where $b<a<3 / 5$, we get

$$
\begin{aligned}
\sum_{x \leqq q \leqq x^{m}+h} & -\log \left(1-\frac{1}{q}\right)=\log m+\frac{\exp -\log ^{b} x}{m \log x} \\
+ & O\left\{\frac{\exp -2 \log ^{b} x}{\log x}+O\left(\exp -\log ^{a} x\right)\right\},
\end{aligned}
$$

and this is greater than $\log m$ for all sufficiently large $x$. Again, if we take $h=-x^{m} \exp \left(-\log ^{b} x\right)$ where $b<a<3 / 5$, then 


$$
\begin{aligned}
\sum_{x \leqq q \leq x^{m}+h} & -\log \left(1-\frac{1}{q}\right)=\log m-\frac{\exp \left(-\log ^{b} x\right)}{m \log x} \\
+ & O\left(\frac{\exp \left(-2 \log ^{b} x\right)}{\log x}\right)+O\left(\exp \left(-\log ^{a} x\right)\right),
\end{aligned}
$$

which is less than $\log m$ for all sufficiently large $x$. Hence, if $g(x)$ is the smallest number such that

$$
\sum_{x \leqq q \leqq g(x)}-\log \left(1-\frac{1}{q}\right) \geqq \log m,
$$

then $g(x)=x^{m}+O\left(x^{m} \exp \left(-\log ^{a} x\right)\right)$ for all $a<3 / 5$. Now going back to the relation

$$
2^{r} \phi(n)+2=K n .
$$

This gives, with $m=2^{r} / K$, the result

$$
m+2 / \phi(n)=n / \phi(n) .
$$

Taking $q_{u}$ to be the smallest prime divisor of $n$, let the integer $v$ be defined to be the smallest integer with the property

$$
m<\prod_{i=u}^{v} \frac{q_{i}}{q_{i}-1}
$$

that is,

$$
\sum_{q_{u} \leqq q \leqq q_{v}}-\log \left(1-\frac{1}{q}\right)>\log m .
$$

Then it follows that $n$ must have a prime factor other than $q_{u}$ and at least as large as $q_{v}$. The previous investigation shows that.

$$
q_{v}=q_{u}^{m}+O\left(q_{u}^{m} \exp \left(-\log ^{a}\left(q_{u}^{m}\right)\right)\right),
$$

that is,

$$
q_{v}=q_{u}^{m}+O\left(u^{m} \exp \left(-\log ^{b} u\right)\right) \text { for any } b<a<3 / 5 .
$$

Hence, we have proved the theorem.

\section{REFERENCES}

1. D. H. Lehmer, On Euler's totient function, Bull. Amer. Math. Soc., 38 (1932), 745-751.

2. E. Lieuwens, Do there exist composite numbers $M$ for which $K \phi(M)=M-1$ holds? Niew Archief von Wiskunde (3), 18 (1970), 165-169.

3. J. B. Roser and L. Schoenfeld, Approximate formulas for some functions of prime numbers, Illinois J. Math., 6 (1962), 64-94.

4. Fr. Schuh, Do there exist composite numbers $m$ for which $\phi(m) \mid m-1$ (Dutch), Mathematica Zutphen B, 13 (1944), 102-107. 
5. A. Walfisz, Weylsche Exponential Summen in den neueren Zahlentheorie, Veb Deutscher Verlag der Wissenschaften, Berlin, 1963.

Received May 20, 1971. Part of this research work was done at the University of Missouri during the summer of 1968. T. J. Cook, R. S. Newberry and J. M. Weber (undergraduate students there at that time) helped the author in the calculations which led to Theorem 4 of the paper.

The University of Alberta 


\section{PACIFIC JOURNAL OF MATHEMATICS}

EDITORS

RICHARD ARENS (Managing Editor)

University of California

Los Angeles, California 90024

R. A. Beaumont

University of Washington

Seattle, Washington 98105

J. DugundJI

Department of Mathematics

University of Southern California

Los Angeles, California 90007

D. Gilbarg and J. Milgram

Stanford University

Stanford, California 94305

\section{ASSOCIATE EDITORS}

E. F. BECKENBACH

B. H. NeumanN

F. WOLF

K. YOSHIDA

\section{SUPPORTING INSTITUTIONS}

UNIVERSITY OF BRITISH COLUMBIA CALIFORNIA INSTITUTE OF TECHNOLOGY

UNIVERSITY OF CALIFORNIA

MONTANA STATE UNIVERSITY

UNIVERSITY OF NEVADA

NEW MEXICO STATE UNIVERSITY

OREGON STATE UNIVERSITY

UNIVERSITY OF OREGON

OSAKA UNIVERSITY

\author{
UNIVERSITY OF SOUTHERN CALIFORNIA \\ STANFORD UNIVERSITY \\ UNIVERSITY OF TOKYO \\ UNIVERSITY OF UTAH \\ WASHINGTON STATE UNIVERSITY \\ UNIVERSITY OF WASHINGTON \\ $\stackrel{*}{*} \stackrel{*}{*}{ }^{*}{ }^{*}{ }^{2}$ AMERICAN MATHEMATICAL SOCIETY \\ NAVAL WEAPONS CENTER
}




\section{Pacific Journal of Mathematics}

\section{Vol. 52, No. $1 \quad$ January, 1974}

David R. Adams, On the exceptional sets for spaces of potentials ............ 1

Philip Bacon, Axioms for the Čech cohomology of paracompacta ............ 7

Selwyn Ross Caradus, Perturbation theory for generalized Fredholm operators ..... 11

Kuang-Ho Chen, Phragmén-Lindelöf type theorems for a system of nonhomogeneous equations ............................ 17

Frederick Knowles Dashiell, Jr., Isomorphism problems for the Baire classes .......

M. G. Deshpande and V. K. Deshpande, Rings whose proper homomorphic images are right subdirectly irreducible . . . . . . . . . . . . . . . . . . . . . . . . .

Mary Rodriguez Embry, Self adjoint strictly cyclic operator algebras .............

Paul Erdős, On the distribution of numbers of the form $\sigma(n) / n$ and on some related

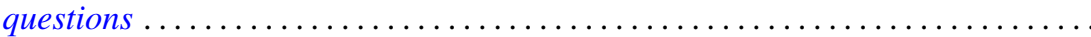

Richard Joseph Fleming and James E. Jamison, Hermitian and adjoint abelian

operators on certain Banach spaces ............................

Stanley P. Gudder and L. Haskins, The center of a poset .................. 85

Richard Howard Herman, Automorphism groups of operator algebras . . . ........

Worthen N. Hunsacker and Somashekhar Amrith Naimpally, Local compactness of families of continuous point-compact relations ....................

Donald Gordon James, On the normal subgroups of integral orthogonal groups ....

Eugene Carlyle Johnsen and Thomas Frederick Storer, Combinatorial structures in

loops. II. Commutative inverse property cyclic neofields of prime-power

order.

Ka-Sing Lau, Extreme operators on Choquet simplexes . . . . . . . . . . . . . . 129

Philip A. Leonard and Kenneth S. Williams, The septic character of 2, 3, 5 and $7 \ldots 143$

Dennis McGavran and Jingyal Pak, On the Nielsen number of a fiber map ........ 149

Stuart Edward Mills, Normed Köthe spaces as intermediate spaces of $L_{1}$ and

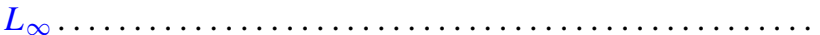

Philip Olin, Free products and elementary equivalence. .

Louis Jackson Ratliff, Jr., Locally quasi-unmixed Noetherian rings and ideals of the principal class.

Seiya Sasao, Homotopy types of spherical fibre spaces over spheres ...

Helga Schirmer, Fixed point sets of polyhedra ...

Kevin James Sharpe, Compatible topologies and continuous irreducible

representations.

Frank Siwiec, On defining a space by a weak base . . . . . . . . . . . . . . . 233

James McLean Sloss, Global reflection for a class of simple closed curves ....... 247

M. V. Subba Rao, On two congruences for primality . .

Raymond D. Terry, Oscillatory properties of a delay differential equation of even

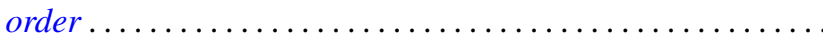

Joseph Dinneen Ward, Chebyshev centers in spaces of continuous functions . .

Robert Breckenridge Warfield, Jr., The uniqueness of elongations of Abelian

groups...

V. M. Warfield, Existence and adjoint theorems for linear stochastic differential

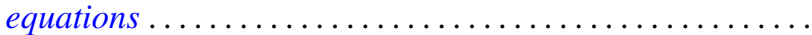

